\title{
Insights from Europe related to pandemic influenza A(H1N1)2009 have international relevance
}

H A Kelly (Heath.Kelly@mh.org.au)1, B J Cowling²

1. Victorian Infectious Diseases Reference Laboratory, 10 Wreckyn St, North Melbourne, Victoria, Australia, 3051

2. School of Public Health, the University of Hong Kong, Hong Kong Special Administrative Region, China

In this issue of Eurosurveillance Amato Gauci and colleagues collate a summary of surveillance data related to pandemic influenza $A\left(\mathrm{H}_{1} \mathrm{~N}_{1}\right)_{2009}$ from the 27 European Union Member States plus Norway and Iceland [1]. While much has already been published on experiences of individual countries, this report is an important summary of the impact of the first influenza pandemic of the 21st century in Europe as a whole. The authors acknowledge the inherent difficulties in summarising data collected from countries with varying surveillance systems and where the pandemic had differential impact. For instance, it was only in England - and only there in London and the West Midlands - that there was a significant spring pandemic wave in 2009 [2]. Like many aspects of the pandemic, this observation remains unexplained.

From a summary of the epidemiological and virological data, the authors recapitulate features of the pandemic that are now generally accepted (Box). However many of these features were not recognised early when an informed understanding was critical to an appropriate pandemic response. For instance, the authors quote a report from the World Health Organization published in 2009 that suggested early estimates of the effective reproduction number $(R)$, defined as the average number of secondary infections attributable to one infectious case, were in the range 1.1-1.4 for the United Kingdom (UK) at the start of the pandemic, although up to 2.6 elsewhere [3]. Only the lower estimates for $\mathrm{R}$ are supported by recent studies [4]. Early estimates of $R$ may have been overestimated for a number of reasons [5]. Firstly, ignoring imported cases or counting imported cases as locally acquired could increase the estimated R. Secondly, early estimates of $R$ based on outbreaks could be overestimated due to selection bias. Thirdly, many early estimates of $\mathrm{R}$ reflected a high proportion of cases among school-age children, amongst whom $\mathrm{R}$ was higher than in the general population [3]. Finally, R could have been overestimated if transmission occurring prior to testing was not recognised [6].
The consensus estimates for $R$ are now similar to those accepted for seasonal influenza [1], suggesting similar transmissibility for both viruses. While early outbreak investigations in schools or households, such as the UK First Few Hundred initiative [7], have the potential to provide timely data on the transmissibility characteristics of a new virus, further work is needed to clarify the extrapolation of transmissibility from outbreak studies to implications for population epidemiology.

\section{Box}

Generally accepted understanding of the 2009 influenza pandemic

- The highest cumulative incidence of disease was in the 0-4 year old age group, although the highest cumulative incidence of infection (including asymptomatic infection) was in school-aged children, the age group which was instrumental in the spread of the pandemic.

- Deaths associated with virologically confirmed influenza were lower than the number of excess deaths thought to occur from seasonal influenza, but the majority of deaths from pandemic influenza $\mathrm{A}\left(\mathrm{H}_{1} \mathrm{~N}_{1}\right) 2009$ occurred at a younger age than is typically seen with seasonal influenza. However excess mortality and laboratory-confirmed deaths are not directly comparable.

- Although older adults were affected less commonly, this was the age group with the highest case fatality ratio.

- Intensive care units were stressed by the increase in the number of young adults with severe disease due to pandemic influenza $A\left(\mathrm{H}_{1} \mathrm{~N}_{1}\right) 2009$, a phenomenon first recognised in the southern hemisphere (19) but not experienced in all countries.

- Pregnant and post-partum women and indigenous people, both recognised risk groups for infection with seasonal influenza, were at apparently increased risk for a severe outcome from pandemic influenza $A\left(\mathrm{H}_{1} \mathrm{~N}_{1}\right)_{2009}$ infection.

- Although pandemic influenza $A\left(\mathrm{H}_{1} \mathrm{~N}_{1}\right) 2009$ appears to have completely replaced previous seasonal influenza $A\left(\mathrm{H}_{1} \mathrm{~N}_{1}\right)$ subtypes, it has not replaced influenza $A\left(\mathrm{H}_{3} \mathrm{~N}_{2}\right)$ subtypes which have continued to co-circulate as a small proportion of all typed influenza A viruses. This contrasts with the observations from previous pandemics, when the pandemic virus replaced all influenza A viruses.

- Unlike the pattern for seasonal influenza $A\left(\mathrm{H}_{1} \mathrm{~N}_{1}\right)$ viruses, no significant neuraminidase resistance of pandemic influenza $A\left(\mathrm{H}_{1} \mathrm{~N}_{1}\right) 2009$ has been detected to date, although variants with reduced oseltamivir sensitivity may be emerging in the Asia-Pacific region [20].

- The pandemic virus was less virulent than had been anticipated in many pandemic plans. 
In trying to further disentangle the comparison of pandemic influenza $A\left(\mathrm{H}_{1} \mathrm{~N}_{1}\right) 2009$ and seasonal influenza in the community, the authors have re-examined data from sentinel surveillance schemes that were operating in Europe during the pandemic and shown that influenza-like illness (ILI) rates were higher during the pandemic than during the previous influenza season (Figure 1 in reference 1). However it is generally acknowledged that the pandemic was associated with increased testing for influenza as well as potential changes in healthcare-seeking behaviour [8]. The proportion of ILI patients who test positive for influenza can be a useful method for comparing influenza seasons, as it can potentially adjust for differential testing between jurisdictions and across seasons [9]. When the metric of percentage positive tests was applied to the European surveillance data, the predominantly pandemic season of $2009 / 10$ looked similar in magnitude to the preceding 2008/9 influenza season (Figure 2 in reference 1 ).

Comparing ILI rates for pandemic and seasonal influenza is a specific example of a more general problem with influenza epidemiology - the extent to which common things are unknown. Further evidence of this problem is provided in the European review when it is suggested that asymptomatic infection was more common for pandemic influenza $A\left(\mathrm{H}_{1} \mathrm{~N}_{1}\right)_{2009}$ than for seasonal influenza, an observation based on admittedly weak evidence [1]. While around one third of experimental infections with a range of influenza types and sub-types are asymptomatic [10], this proportion depends on the definition of asymptomatic infection. Prospective intensive follow-up of people in household studies has found that only around $10 \%$ of virologically-confirmed $\mathrm{A}\left(\mathrm{H}_{1} \mathrm{~N}_{1}\right)_{2009}$ infections were completely asymptomatic, while around one half were associated with febrile illness [11-13]. The precise asymptomatic fraction of naturally acquired infections due to seasonal and pandemic influenza remains uncertain, as does the potential for variability in this fraction by age.

Trying to understand the pandemic in Europe and around the world has highlighted other uncertainties about influenza epidemiology.

- Except for infants and children aged 0-4 years, for whom routine laboratory testing is common in many places, the number of hospitalisations due to laboratory-confirmed influenza is poorly estimated for other age groups. This number will vary by year, and by influenza type and subtype. The proportion of those requiring admission to intensive care will also vary by these parameters.

- Similarly, the number of deaths that can be directly attributed to laboratory-confirmed influenza is not known for the same parameters. Although underestimated, the increased testing associated with the pandemic provided estimates of laboratory confirmed deaths, but generally only for $\mathrm{A}\left(\mathrm{H}_{1} \mathrm{~N}_{1}\right)_{2009}$ infections.
- Controversy persists over estimates of excess deaths attributable to influenza. These estimates place a substantial burden of seasonal influenza on the elderly and are not directly comparable to estimates of virologically confirmed deaths. Although estimates of years of life lost have been made, these have not yet been adjusted for the presence of preexisting conditions.

- The proportion of people with confirmed influenza who seek medical attention is poorly understood in most countries. This proportion is very likely to reflect differences in cultural attitudes to illness, the provision of medical services and the public health interventions implemented in different countries. Serologic studies in combination with outpatient and inpatient surveillance can improve these estimates $[14,15]$.

- There are very limited published data on the proportion of people with naturally acquired laboratory-confirmed influenza whose infections are asymptomatic. The likelihood of transmission from people with asymptomatic infections to susceptible contacts is not known.

- Vaccine is known to be effective in healthy children and adults but vaccine effectiveness is poorly understood in the elderly and in individuals at higher risk of severe disease if infected. These are the groups targeted for vaccination $[1,16]$.

- Influenza usually circulates in the winter in temperate settings, but was able to spread in the spring in some parts of Europe and North America, raising questions about the diverse causes of influenza seasonality.

Three of the highlighted recommendations made by Amato Gauci and colleagues reflect the importance of filling these gaps in our knowledge of influenza epidemiology [1]:

Firstly, they recommend making 'severe end' influenza surveillance routine. Routine community-based influenza surveillance was very useful during the pandemic and routine hospital-based surveillance ('severe end' surveillance) would have been equally useful. A study from Australia suggested that the hospital course for adults was similar for those infected with pandemic influenza $A\left(\mathrm{H}_{1} \mathrm{~N}_{1}\right)_{2009}$ and those infected with seasonal influenza - but that the burden on the hospital system resulted from the increased number of adults admitted to hospital during the pandemic [17]. Uncertainties surround this issue because of the lack of quality surveillance data from hospitals over a number of influenza seasons [18].

Secondly, they recommend sharing data early in any future outbreak. Data sharing facilitated international attempts to gauge the severity of the pandemic in 2009. This undertaking was supported by the unique rapid peer-reviewed publication policy of Eurosurveillance. The accuracy of shared articles was less certain when rapid publication dispensed with peer-review. 
Thirdly, they suggest that sero-epidemiological studies should be included in revised pandemic plans to provide information in real time. This may be the most optimistic of the recommendations [15]. Serological studies remain the best approach to estimate the cumulative incidence of infection following a wave of infection but technical issues remain unsolved. These include the correlation between antibody titres and immunity, the characteristics of antibody profiles over time, the potential effect of antiviral treatment on convalescent antibody [11], and the interpretation of serological data after the introduction of a vaccine. The use of serological data for real-time evaluation of severity also requires reliable surveillance of severe infections [14].

Many aspects of improved understanding require descriptive and analytical epidemiological studies in diverse countries over consecutive influenza seasons in order to capture the range of potential outcomes due to laboratory-confirmed influenza, the outcome of choice in attempting to understand influenza control measures [16]. This level of understanding appears to be long overdue and should not be deferred until the next pandemic.

\section{Acknowledgments}

We thank the following people for their comments on the manuscript: Dr Aeron Hurt from the WHO Collaborating Centre for Surveillance and Research on Influenza, Melbourne, Australia; and Dr Geoffry Mercer and Dr Kathryn Glass from the Australian National University, Canberra, Australia. BJC is supported by supported by the Harvard Center for Communicable Disease Dynamics from the US National Institutes of Health Models of Infectious Disease Agent Study program (grant number 1 U54 GMo88558). BJC has received research funding from MedImmune Inc. HK has received funding from CSL Biotherapies. The authors report no other potential conflicts of interest. The views expressed are those of the authors.

\section{References}

1. Amato-Gauci A, Zucs P, Snacken R, Ciancio B, Lopez V, Broberg $E$, et al. Surveillance trends of the 2009 influenza $A\left(\mathrm{H}_{1} \mathrm{~N}_{1}\right)$ pandemic in Europe. Euro Surveill. 2011;16(26):pii=19903. Available from: http://www.eurosurveillance.org/ViewArticle. aspx?Articleld $=19903$

2. Flasche S, Hens N, Boelle PY, Mossong J, van Ballegooijen WM, Nunes $B$, et al. Differential transmission patterns in the early stages of the influenza $A\left(\mathrm{H}_{1} \mathrm{~N}_{1}\right)$ v pandemic: a comparative analysis of 12 European countries. Epidemics. 2011;3(2):125-33.

3. World Health Organization (WHO). Transmission dynamics and impact of pandemic influenza A ( $\left.\mathrm{H}_{1} \mathrm{~N}_{1}\right) 2009$ virus. Wkly Epidemiol Rec. 2009;84(46): 481-4.

4. Nishiura H, Chowell G, Castillo-Chavez C. Did modeling overestimate the transmission potential of pandemic (H1N1-2009)? Sample size estimation for post-epidemic seroepidemiological studies. PLoS One 2011;6(3):e17908.

5. Mercer G, Glass K, Beckers N. Effective reproduction numbers are commonly overestimated early in a diseases outbreak. Stat Med. 2011;30(9):984-94

6. Kelly H, Mercer G, Fielding J, Dowse G, Glass K, Carcione D, et al. Community circulation of pandemic influenza $\mathrm{H}_{1} \mathrm{~N}_{1}$ was established in one Australian state at the same time the virus was first recognised in North America. PloS One 2010;5(6):e11341.
7. Pebody RG, Harris R, Kafatos G, Chamberland M, Campbell C, Nguyen-Van-Tam JS, et al. Use of antiviral drugs to reduce household transmission of pandemic $\left(\mathrm{H}_{1} \mathrm{~N}_{1}\right) 2009$, United Kingdom. Emerg Infect Dis. 2011;17(6).

8. Miller E, Hoschler K, Hardelid P, Stanford E, Andrews N, Zambon M. Incidence of 2009 pandemic influenza $\mathrm{A} \mathrm{H}_{1} \mathrm{~N}_{1}$ infection in England: a cross-sectional serological study. Lancet. 2010;375(9720):1100-8.

9. Lambert SB, Faux CE, Grant KA, Williams SH, Bletchly C, Catton MG et al. Influenza surveillance in Australia: we need to do more than count. Med J Aust. 2010;193(1):43-5.

10. Carrat F, Vergu E, Ferguson NM, Lemaitre M, Cauchemez S, Leach, et al. Time lines of infection and disease in human influenza: a review of volunteer challenge studies. Am J Epidemiol. 2008;167(7):775-85.

11. Cowling BJ, Chan KH, Fang VJ, Lau LL, So HC, Fung RO, et al. Comparative epidemiology of pandemic and seasonal influenza A in households. N Engl J Med. 2010;362(23):2175-84.

12. Suess T, Buchholz U, Dupke S, Grunow R, an der Heiden M, Biere $B$, et al. Shedding and transmission of novel influenza virus $A / H_{1} N_{1}$ infection in households--Germany, 2009. Am J Epidemiol. 2010;171(11):1157-64.

13. Papenburg J, Baz $M$, Hamelin MÈ, Rhèaume $C$, Carbonneau J, Ouakki M, et al. Household transmission of the 2009 pandemic $\mathrm{A} / \mathrm{H}_{1} \mathrm{~N}_{1}$ influenza virus: elevated laboratory-confirmed secondary attack rates and evidence of asymptomatic infections. Clin Infect Dis. 2010;51(9):1033-41.

14. Wu JT, Ho A, Ma ES, Lee CK, et al. Estimating infection attack rates and severity in real-time during an influenza pandemic. PLoS Med. 2011; forthcoming.

15. Kelly H, Peck H, Laurie K, Wu P, Nishiura H, Cowling BJ. The age specific cumulative incidence of infection with pandemic influenza $\mathrm{H}_{1} \mathrm{~N}_{1} 2009$ was similar in various countries prior to vaccination: a review of serological surveys. PloS One. 2011; forthcoming.

16. Valenciano M, Kissling E, Cohen J M, Oroszi B, Barret AS, Rizzo $C$, et al. Estimates of pandemic influenza vaccine effectiveness in Europe, 2009-2010: Results of Influenza Monitoring Vaccine Effectiveness in Europe (I-MOVE) multicentre case-control study. PLoS Med. 2011;8(1):e1000388.

17. Chang YS, van Hal SJ, Spencer PM, Gosbell IB, Collett PW. Comparison of adult patients hospitalised with pandemic $\left(\mathrm{H}_{1} \mathrm{~N}_{1}\right) 2009$ influenza and seasonal influenza during the "PROTECT" phase of the pandemic response. Med J Aust. 2010;192(2):90-3.

18. Kelly PM, Kotsimbos T, Reynolds A, Wood-Baker R, Hancox B, Brown SG, et al. FluCAN 2009: initial results from sentinel surveillance for adult influenza and pneumonia in eight Australian hospitals. Med J Aust. 2011;194(4):169-74.

19. ANZIC Influenza Investigators, Webb SA, Pettilä V, Seppelt I, Bellomo R, Bailey M, et al. Critical care services and 2009 $\mathrm{H}_{1} \mathrm{~N}_{1}$ influenza in Australia and New Zealand. N Engl J Med. 2009;361(20):1925-34.

20. Hurt AC, Lee RT, Leang SK, Cui L, Deng Y, Phuah S, et al. Increased detection in Australia and Singapore of a novel influenza $\mathrm{A}\left(\mathrm{H}_{1} \mathrm{~N}_{1}\right) 2009$ variant with reduced oseltamivir and zanamivir sensitivity due to a $\mathrm{S}_{47} \mathrm{~N}$ neuraminidase mutation. Euro Surveill. 2011;16(23):pii=19884. Available from: http:// www.eurosurveillance.org/ViewArticle.aspx?Articleld =19884 